\title{
Caso radiológico de desafío diagnóstico: Distensión y dolor abdominal
}

Dres. Andrés Retamal C(1), Matías Molina $V^{(1)}$, Claudio Cortés ${ }^{(2)}$, Giancarlo Schiappacasse $F^{(2)}$.

1. Residente Programa Especialidad en Radiología. Facultad de Medicina Clínica Alemana Santiago.

Universidad del Desarrollo, Santiago, Chile.

2. Médico Radiólogo Clínica Alemana Santiago, Chile.

\section{Historia clínica}

Paciente de sexo femenino de 74 años, con antecedente de cirugía de colon hace 10 años, de la cual no se cuenta con mayores antecedentes. Consulta en servicio de urgencia por distensión y dolor abdominal de siete días de evolución que en los últimos dos días se asocia a la ausencia de deposiciones y eliminación de gases. Se solicita radiografía $(\mathrm{Rx})$ de abdomen simple (Figura 1).

¿Cuál es su diagnóstico?
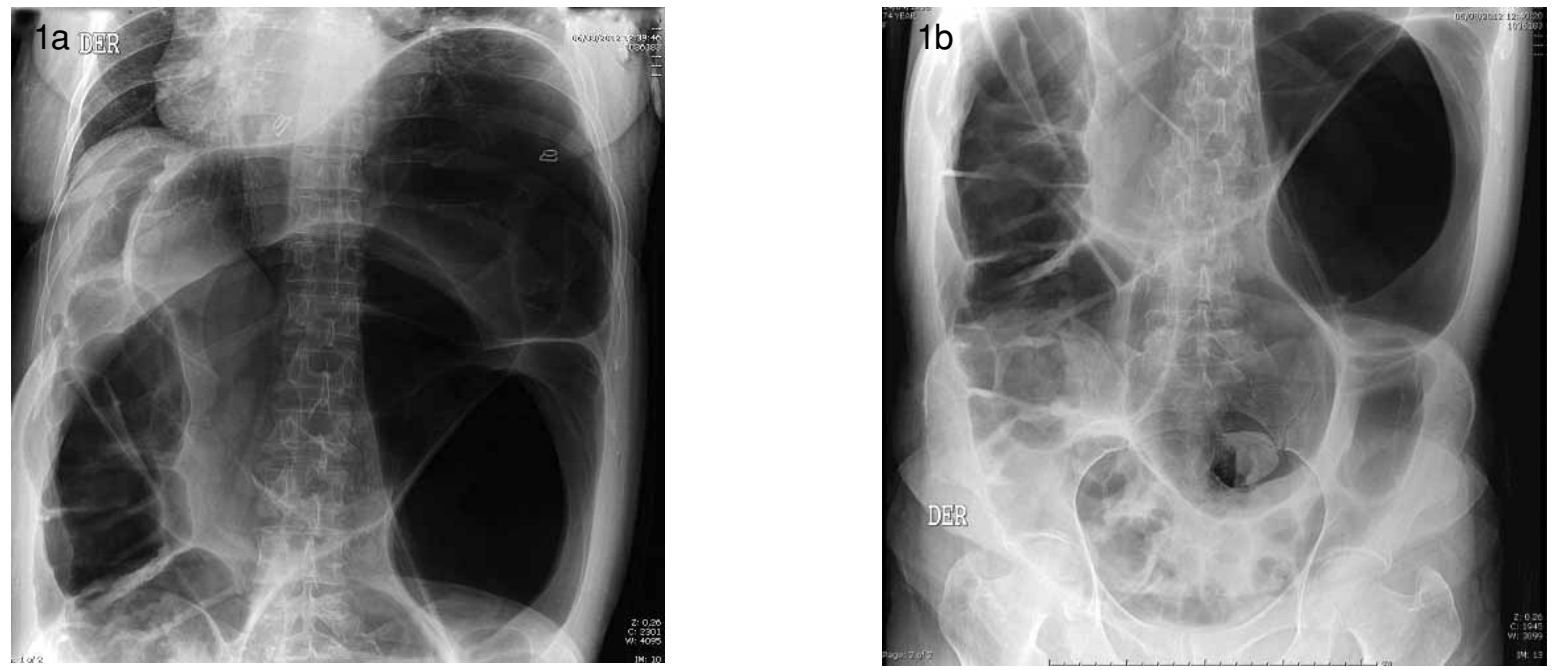

Figura 1a,b. 


\title{
Resultado caso radiológico de desafío diagnóstico
}

\author{
Dres. Andrés Retamal $C^{(1)}$, Matías Molina $V^{(1)}$, Claudio Cortés $A^{(2)}$, Giancarlo Schiappacasse $F^{(2)}$.
}

1. Residente Programa Especialidad en Radiología.

Facultad de Medicina Clínica Alemana Santiago - Universidad del Desarrollo, Santiago, Chile.

2. Médico Radiólogo Clínica Alemana Santiago, Chile.

viene de la página 96

\section{Diagnóstico}

Íleo biliar con obstrucción del colon

\section{Hallazgos}

En la Rx de abdomen simple se observa importante dilatación del marco cólico, cuyas asas alcanzan hasta $20 \mathrm{~cm}$. de diámetro mayor (Figura 1) visualizándose además en la excavación pelviana una imagen con densidad cálcica de aproximadamente $5 \mathrm{~cm}$ de diámetro mayor; con borde redondeado y un segmento irregular que se orienta hacia el lumen del recto hacia distal (Figura 2).
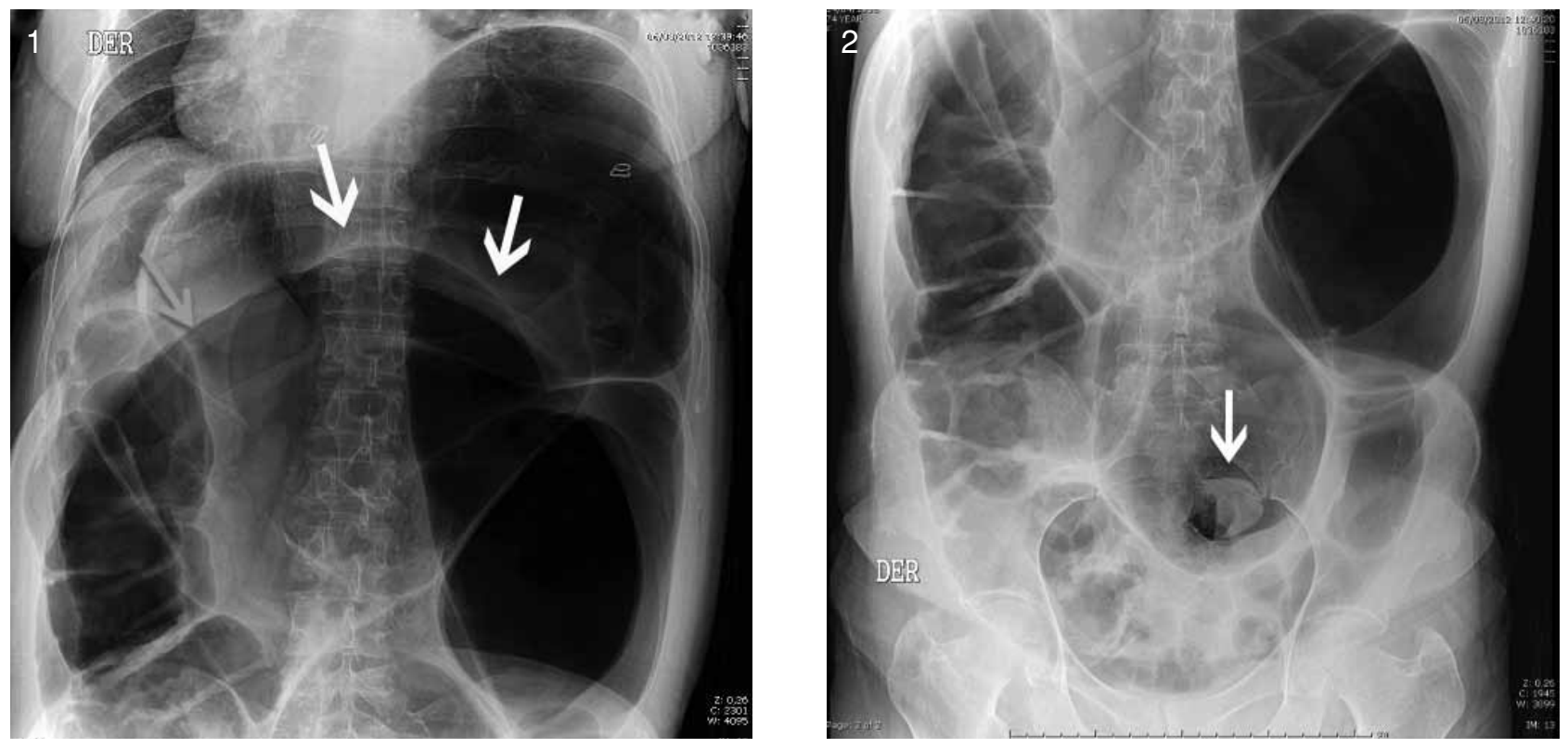

Se complementa estudio con tomografía computada (TC) de abdomen y pelvis.

En la TC no contrastada se demuestra la presencia de aire en la vía biliar (neumobilia) e importante dilatación del marco cólico. Las asas dilatadas se ubican en intimo contacto con la pared abdominal, la que además presenta alteraciones secundaria a una hernia postoperatoria. Se identifica también una estructura calcificada compatible con un calculo biliar. Este se encuentra impactado adyacente a zona de resección quirúrgica previa, generando una obstrucción mecánica en forma secundaria (Figura 3).

Debido a la localización del cálculo, se realizó rectoscopia y extracción por vía manual en pabellón con anestesia epidural. Posterior al procedimiento presenta importante mejoría clínica y desaparición de la distensión abdominal en la inspección (Figura 4).

Figuras 1 y 2. 

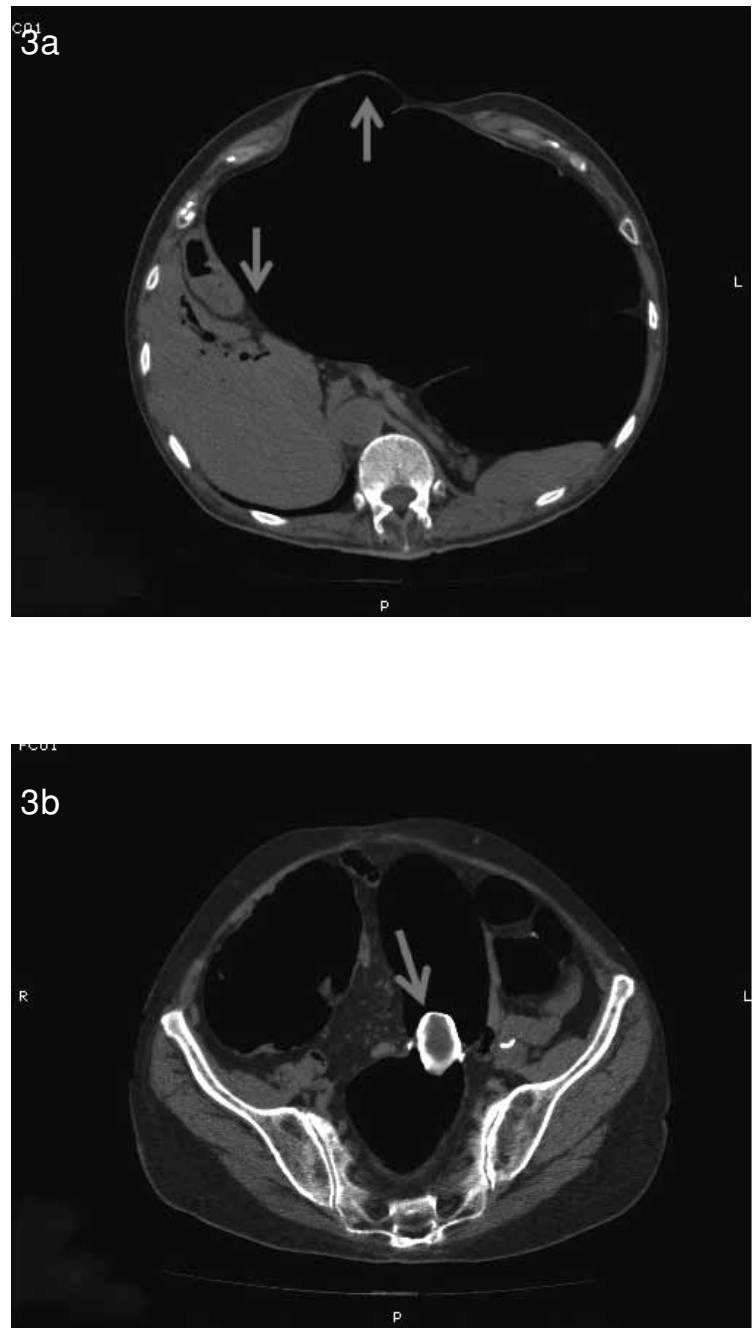

Figura 3a,b.

\section{Discusión}

La obstrucción de colon ocurre en aproximadamente un $2-4 \%$ de los íleos biliares ${ }^{(1,2)}$ y es secundaria a migración del cálculo que se impacta en general en una estenosis pre-existente, de origen neoplásica, quirúrgica o enfermedad diverticular.

Descrito en 1654 por Bartholin, el íleo biliar (IB) se define como la obstrucción mecánica del tubo digestivo producida por el impacto de un cálculo en la luz intestinal. Generalmente el cálculo debe medir más de $2,5 \mathrm{~cm}$ para producir obstrucción ${ }^{(2)}$.

EI IB es una complicación rara de la litiasis biliar que ocurre en apenas el $0,3-0.5 \%$ de estos pacientes y supone el $1-6 \%$ del total de las obstrucciones intestinales mecánicas del adulto(3).

La condición esencial para el íleo biliar es la presencia de una fístula entre la vesícula biliar y el tracto gastrointestinal. Con frecuencia se precede de un cuadro de colecistitis aguda y existen antecedentes biliares de larga data en 25 a $75 \%$ de los casos clínicos de cuadros, pero también se encuentra en pacientes sin historia de enfermedad biliar ${ }^{(4)}$.

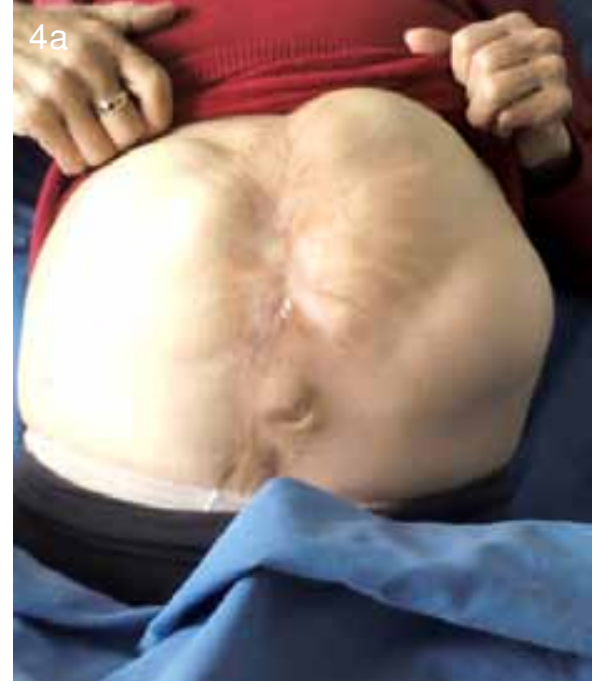

Figura 4a. Fotografía clínica previo al procedimiento, donde destaca distensión abdominal.

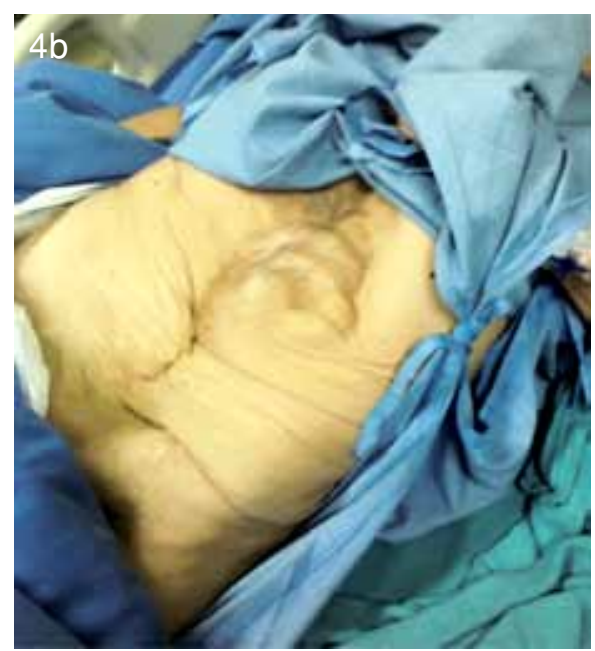

Figura 4b. Fotografía clínica durante procedimiento, posterior a extracción.

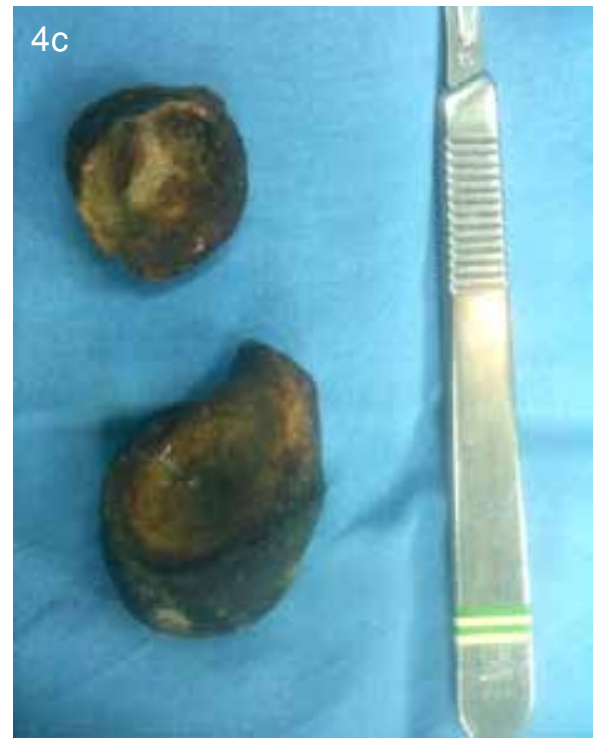

Figura 4c. Cálculo extraído, de $5 \mathrm{~cm}$ de diámetro mayor. 
Es más frecuente en ancianos y la mayoría de las series nacionales e internacionales demuestran un promedio de edad entre 65 y 75 años, afectando principalmente al sexo femenino(3).

El cuadro clínico más común es una obstrucción intestinal mecánica con distensión, dolor abdominal, vómito y constipación.

Los hallazgos clásicos de la Rx de abdomen simple corresponden a la tríada de Rigler: distensión intestinal, neumobilia y cálculo extravesicular, presente en sólo en un $30-35 \%$ de los $\operatorname{casos}^{(5,6)}$. En ecografía, la presencia de neumobilia y vesícula esclero-atrófica son sugerentes de fístula bilio-digestiva.

La TC de abdomen es el método de elección para confirmar el diagnóstico, con una sensibilidad reportada de un $93 \%$ y una especificidad de $100 \%{ }^{(7)}$; permite la detección de litiasis única o múltiple en el tubo digestivo así como complicaciones asociadas y también permite detectar la causa y el punto de obstrucción(6). Los hallazgos tomográficos pueden incluir la visualización de un cálculo de ubicación ectópica, neumobilia y paredes vesiculares engrosadas, irregulares o con niveles hidro-aéreos en su lumen ${ }^{(7)}$.

La TC entrega con gran exactitud el diámetro y la localización del cálculo, lo que permite elegir la forma de tratamiento más apropiada.

El uso de contraste endovenoso en TC, se recomienda principalmente para establecer un adecuado diagnóstico diferencial y también para detectar de mejor manera las complicaciones asociadas ${ }^{(6)}$.
El tratamiento es esencialmente quirúrgico. El manejo médico es conservador hasta en un $12 \%$ de los casos, en algunas series. El diagnóstico de íleo biliar sigue siendo un desafío y sólo cerca de $43 \%$ de los casos es identificado antes de la intervención quirúrgica ${ }^{(8)}$.

\section{Bibliografía}

1. Osman N, Subar D, Loh Mong-Yang. Gallstone lleus of the Sigmoid Colon: An Unusual Cause of Large-Bowel Obstruction. HPB Surg 2010; 2010: 153740.

2. Lassandro F, Romano S, Ragozzino A, Rossi G, Valente T, Ferrara I, et al. Role of Helical CT in Diagnosis of Gallstone lleus and Related Conditions. AJR November 2005: 185; 1159-1165.

3. Reisner RM, Cohen JR. Gallstone ileus: a review of 1001 reported cases. Am Surg 1994; 60: 441-446.

4. Pérez A, Pérez D, Calvo M. Acute obstruction of the colon secondary to biliary lithiasis. Rev Esp Enf Dig 1996; 88: 805-808.

5. Balthazar EJ, Schechter LS. Air in gallbladder: afrequent finding in gallstone ileus. AJR Am J Roentgenol 1978; 131: 219-222.

6. Aloysious A, Richardson P, Karanth S Gallstone ileus with classical Rigler's triad. Gastroenterology Today 2007; 17(3): 74-76.

7. Yu CY, Lin CC, Shyu RY, Hsieh CB, Wu HS, Tyan YS et al. Value of $C T$ in the diagnosis and management of gallstone ileus. World J Gastroenterol 2005; 11(14): 2142-2147.

8. Martínez D, Daroca JJ, Escrig J, Paiva G, Alcalde M, Salvador JL. Íleo biliar: opciones terapéuticas y resultados en una serie de 40 casos. Rev Esp Enferm Dig 2009; 101(2): 117-124.

Molina M, et al. Íleo biliar con obstrucción del colon. Rev Chil Radiol 2013; 19(3): 96, 140-142.

Correspondencia: Dr. Matías Molina / matiasmolina@gmail.com

Trabajo recepcionado el 31 de mayo de 2013, aceptado para publicación el 07 de julio de 2013. 